\title{
Numerical simulations of local and global buckling of hyperelastic tubes with different cross-sections
}

\author{
Lukasz Kowalewski ${ }^{1}$, and Stanistaw Jemioło ${ }^{1, *}$ \\ ${ }^{1}$ WUT, The Faculty of Civil Engineering, ul. Lecha Kaczyńskiego 16, 00-637 Warsaw, Poland
}

\begin{abstract}
The aim of the article is to present the application of finite element method (FEM) programs ABAQUS/Standard [1] and ABAQUS/Explicit [2] and the constitutive models of incompressible isotropic hyperelastic materials [3] in the analysis of local and global buckling of axially compressed shell elements made of elastomers. Three FEM models of tubes with the same length and initial stiffness have been created for this purpose. These are tubes with elliptical, square and triangular cross-sections. Three types of constitutive models of a rubberlike (elastomeric) material are used - with the polynomial function of elastic energy in the form of the model MV [3] and standard models of Neo-Hooke and Mooney-Rivlin [4]. No imperfections are introduced in the FEM models of the analyzed pipes. Numerical simulations of buckling of pipes are performed for two types of initial-boundary value problems, i.e. quasi-static and dynamic ones. It has been shown that the type of buckling depends on the cross-section of the pipe. The solutions of buckling of pipes modelling with different constitutive models are compared and good correlations of the results have been observed.
\end{abstract}

\section{Introduction}

Elastomers are commonly used in civil engineering as well as structural shell elements, in particular pipes of different cross-sections. The aim of the article is to present the application of the ABAQUS finite element software [1,2] with multiple constitutive relations of incompressible isotropic hyperelastic materials in the analysis of compressed and stretched shell elements made of elastomers. For this purpose, three models of thinwalled bars with different cross-sections are presented. These are the tubes with elliptical, square and triangular cross-sections. The latter two cross-sections are created with rounded corners, as to avoid the phenomenon of stress concentration. Additionally, three types of models of a rubber-like material are used - with the polynomial function of elastic energy in the form of the MV [3] model, and the models of Neo-Hooke and Mooney-Rivlin [4]. The FEM jobs (stretching and compression of tubes) presented in this work can be divided into two types in terms of analysis, i.e. static tasks and quasi-static tasks. Static tasks are solved using the Implicit method and the ABAQUS / Standard [1] solver, while the quasi-static

\footnotetext{
* Corresponding author: $\underline{\text { s.jemiolo@il.pw.edu.pl }}$
} 
tasks arehandled by the Explicit method using the ABAQUS / Explicit [2] solver. In the latter case, due to the buckling phenomenon, the analysis can be treated as quasi-static only up to a certain point, as the sudden buckling makes it impossible to omit dynamic phenomena.

The presented tasks enforced no imperfections at the created models. For the FEM calculations, the ABAQUS/Standard program assumes that the loads are proportional, i.e. all load quantities differ only by a single scalar parameter. Because the shells are loaded via displacement boundary conditions, the standard Newton-Raphson method is used, which is the basic algorithm used for nonlinear boundary value problems [5-7].

In works [3,5], for similar examples of compression of hyperelastic tubes with circular and elliptical cross-section, a 3D approach in FEM modelling was used. In order to compare both approaches, in addition to shell one, a three-dimensional model of an elliptical tube is analysed, where elements with parabolic shape functions and the displacement-stress hybrid formulation are used.

\section{Finite element models}

The constitutive relation of the material was described as a hyperelastic, incompressible model, and three different strain energy potential formulas are used, all based on [4]:

$$
W=U\left(\bar{I}_{1}, \bar{I}_{2}\right)=\sum_{k+l=1}^{N} C_{k l}\left(\bar{I}_{1}-3\right)^{k}\left(\bar{I}_{2}-3\right)^{l},
$$

Where non-zero constants $C_{k l}$ are given in tab.2.1. The data provided stands for polynomial model (MV) [3], Mooney-Rivlin model (MR) and Neo-Hooke model (NH) [3,4].In the case of $\mathrm{NH}$ and MR models the material parameters are derived based on the polynomial model parameters in such a way, that the initial material stiffness are the same in every case.

Table 1. Non-zero constants for the analysed material models.

\begin{tabular}{|l|l|l|l|l|l|}
\hline Model & $C_{10}[\mathrm{MPa}]$ & $C_{01}[\mathrm{MPa}]$ & $C_{20}[\mathrm{MPa}]$ & $C_{11}[\mathrm{MPa}]$ & $C_{30}[\mathrm{MPa}]$ \\
\hline MV & 0.179215 & 0.007725 & -0.0018975 & $-9.010^{-5}$ & $4.510^{-5}$ \\
\hline MR & 0.179215 & 0.007725 & - & - & - \\
\hline NH & 0.18694 & - & - & - & - \\
\hline
\end{tabular}

The constraints of the incompressibility of the material, used in ABAQSU/Standard models, cannot be applied for the ABAQUS/Explicit analysis. Because of that, for the jobs calculated with Explicit methods, the material compressibility is assumed in the form of a penalty function included in strain energy potential:

$$
W=U\left(\bar{I}_{1}, \bar{I}_{2}\right)+\frac{1}{D_{10}}\left(J^{2}-1\right),
$$

In the equation above, $D_{10}=0.05[\mathrm{MPa}]$. In addition, the material mass density is assumed as $d=2.0\left[\mathrm{~g} / \mathrm{cm}^{3}\right]$.

Each shell FEM model consists of S4R bilinear shell elements with reduced integration [1], whereas 3D model uses C3D20RH elements -hybrid formulation 20-node elements with quadriatic shape functions $[1,4]$. The cross-sections of the tubes are presented in Figure 1 . The length of each tube equals 200 [mm]. The boundary conditions are applied by displacements for two cases:

a) Same displacement loading of the every node on the extreme cross-section of the bar, where the nodes at the fixed end of the bar had displacements set to zero: $u_{1}=u_{2}=u_{3}=0$, with no constraints on rotations. The other edge is set to: 
$u_{1}=u_{2}=0, u_{3}=U$ where the load is set to $U=800[\mathrm{~mm}]$ in the case of tension, and $U=-50[\mathrm{~mm}]$ for compression.

b) Compression enforced via contact between the analysed pipe and the shell rigid body that transferred the loading onto the bar - in this case the extreme displacement equal $U=-50[\mathrm{~mm}]:$ at the fixed and: $u_{1}=u_{2}=u_{3}=0, \theta_{1}=\theta_{2}=\theta_{3}=0$, and the other end: $u_{1}=u_{2}=0, u_{3}=U, \theta_{1}=\theta_{2}=\theta_{3}=0$.

a)

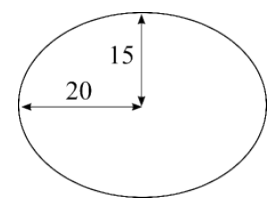

b)

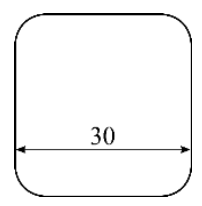

c)

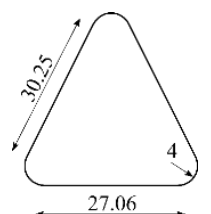

Fig. 1. The cross-sections of the models with dimensions in [mm]. The wall of each cross-section had $2 \mathrm{~mm}$ thickness. The dimensions are assumed so that every model has similar initial stiffness.

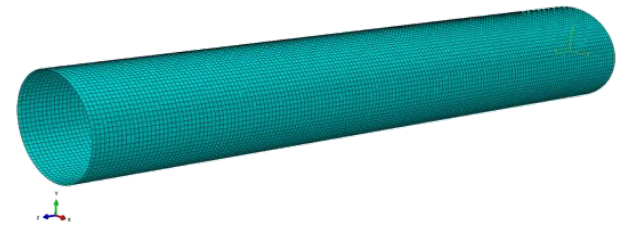

a)

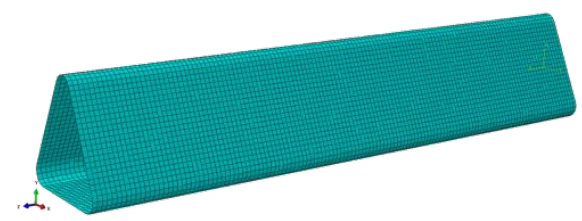

c)

Fig. 2. FEM mesh of the pipe with: a) elliptical cross-section (13992 el. S4R), b) square cross-section $(14080 \mathrm{el} . \mathrm{S} 4 \mathrm{R}), \mathrm{c})$ triangle cross-section (22400 el. S4R) i d) elliptical 3D cross-section (52200 el. C3D20RH).

\section{Presentation of the results}

Each analysis is performed with non-linear geometry (NLGEOM option) enabled. In the case of tensile tests only static ABAQUS/Standard analysis is considered [1], whilst for the compressive tasks such solutions are strongly unstable because of buckling of the tubes. Thus, only Explicit analysis [2] allowed for observation of post-critical behaviour.

For the tube with elliptical cross-section the three-dimensional model is presented as well. Because of relatively small thickness of the cross-section's walls, such approach require greater FE mesh density, which results in general raise in complexity of the numerical calculations. Total variables count in the model increases over 5 times (to around 1000000), and the time and resources required to solve the problem increase an order of magnitude. 
The comparison of numerical solutions is performed mainly by comparing the global reactions of the bar against the displacement boundary conditions. For the compression tests in ABAQUS/Explicit, the analogical reaction is between the rigid shell and deformable bar.

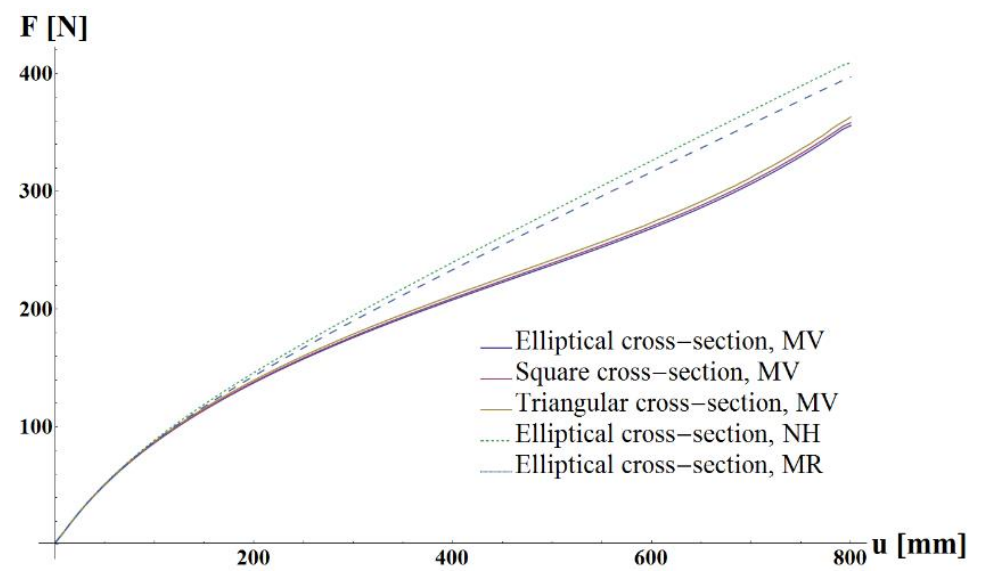

Fig. 3. Comparison of the reaction to tension of the tubes.

Figure 3 presents the comparison between the tensile reaction force between each analysed cross-section and MV material. As the cross sections are assumed with similar areas, those reactions are nearly indistinguishable. On the other hand, the reactions of the samples with different material model showed similarities only in the initial loading phase, with the Neo-Hooke model showing slightly greater stiffness in the late loading phase $(\lambda>2)$. However, the polynomial model would present significant raise in stiffness had the elongation of the sample been even larger. The difference in the range of applicability of each model can be obviously seen - NH and MR models start to deviate from the MV model from the extreme displacement of around $200 \mathrm{~mm}(100 \%$ of the length of the sample).

On the contrary, when comparing the response of the compressed models, no obvious difference can be observed between each material type.

a)

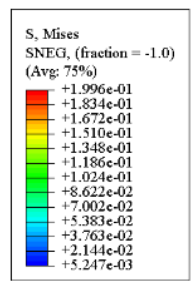

b)

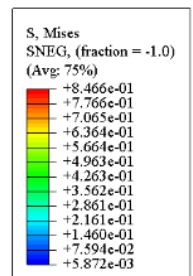

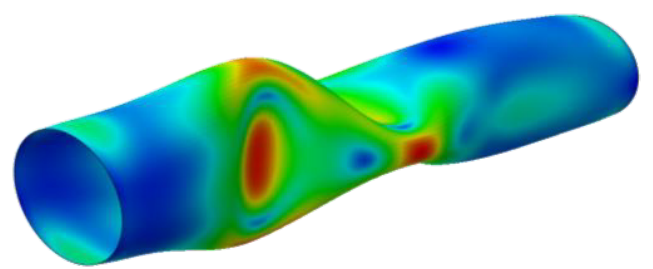

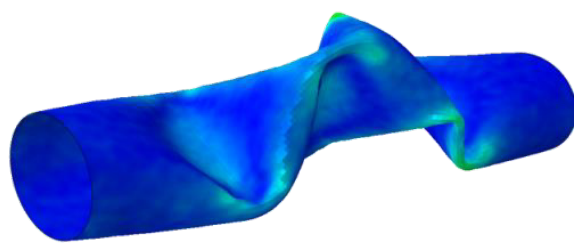

Fig. 4. Buckling mode comparison for the pipe with elliptical cross-section by methods: a) ABAQUS/ Standard and b) ABAQUS/Explicit. 
When analysing the bars, the shell elements where used. However, it can be observed for the elliptical sample, that the 3D model, despite having almost the exact stiffness of the shell model, is losing stability in the later phase of the job than the shell model.

Figures 4-6 present the deformation maps of the samples in the post-buckling phase for Standard and Explicit analyses.

a)
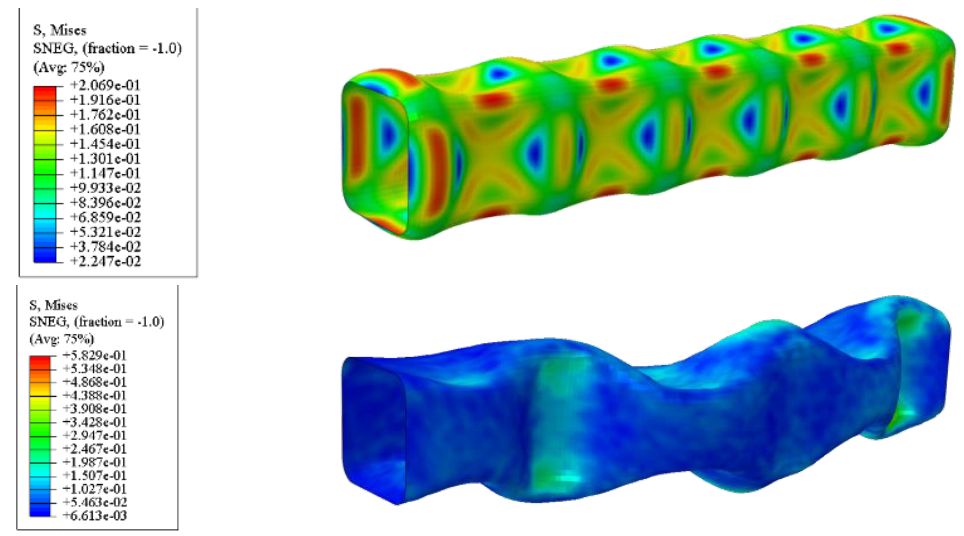

b)

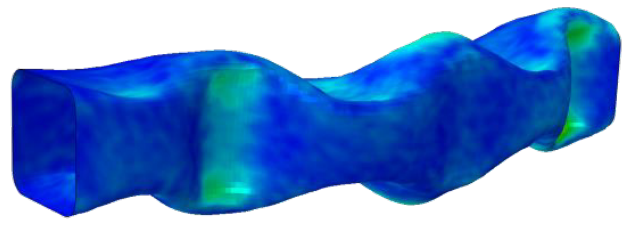

Fig. 5. Buckling mode comparison for the pipe with square cross-section by methods: a) ABAQUS/ Standard and b) ABAQUS/Explicit.

a)
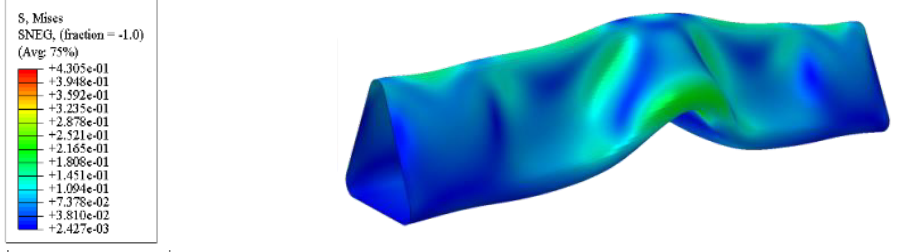

b)
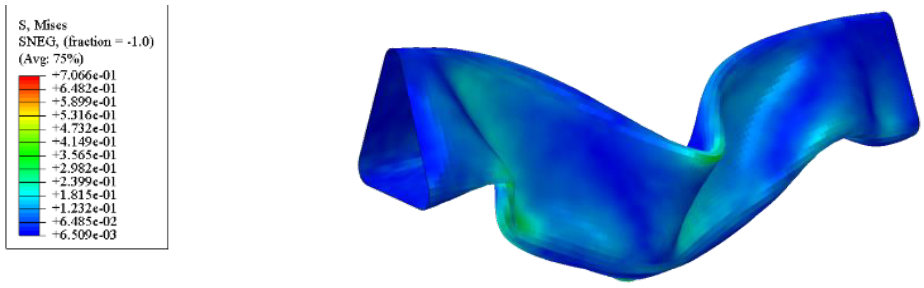

Fig. 6. Buckling mode comparison for the pipe with triangular cross-section by methods: a) ABAQUS/Standard and b) ABAQUS/Explicit.

The comparisons of the reactions of each model for the Explicit and Standard analyses and displacement boundary conditions are presented on Figure 7. It can be observed, that even though the compressibility of the material is assumed in the Explicit analysis, the stiffness of the models coincides with the stiffness in Standard ones. After the stability loss of each sample, the Explicit results show the dynamic vibrations appearing as "noise" in Figures 7 and 8. One can also observe, that the samples analysed by ABAQUS/Explicit show a lower load capacity than the tubes solved statically; however, the stiffness of the samples coincides. Interestingly, in the case of Explicit tasks, the largest load capacity is observed for the bar with elliptical cross-section, whereas in the case of Standard analysis the square cross -section carries greater force (despite the fact that elliptical cross-section 
shows the same stiffness in both analysis types). It is related to the fact that in static task the sample is subjected to local buckling only, as opposed to dynamic task.

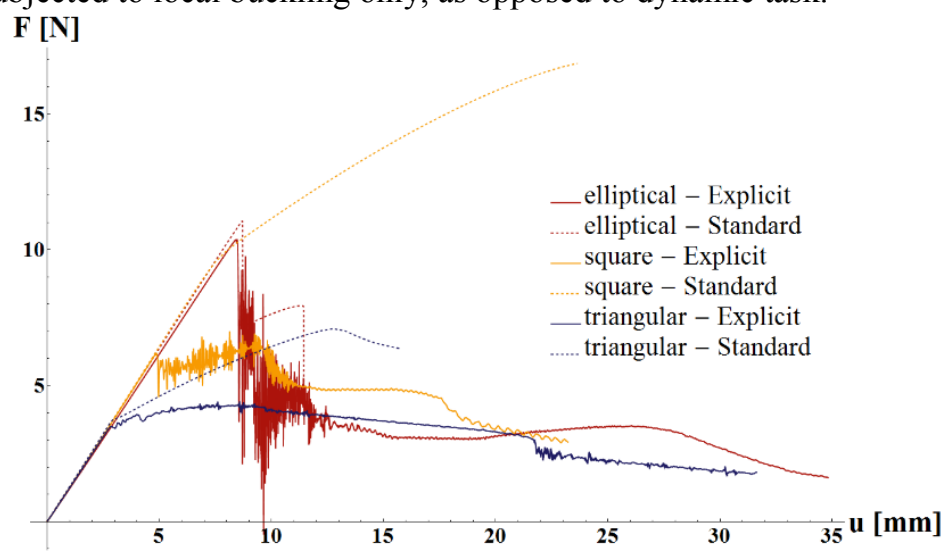

Fig. 7. Comparison of the reaction of the compressed bars with different cross-sections (displacement loading) for Standard and Explicit analysis.

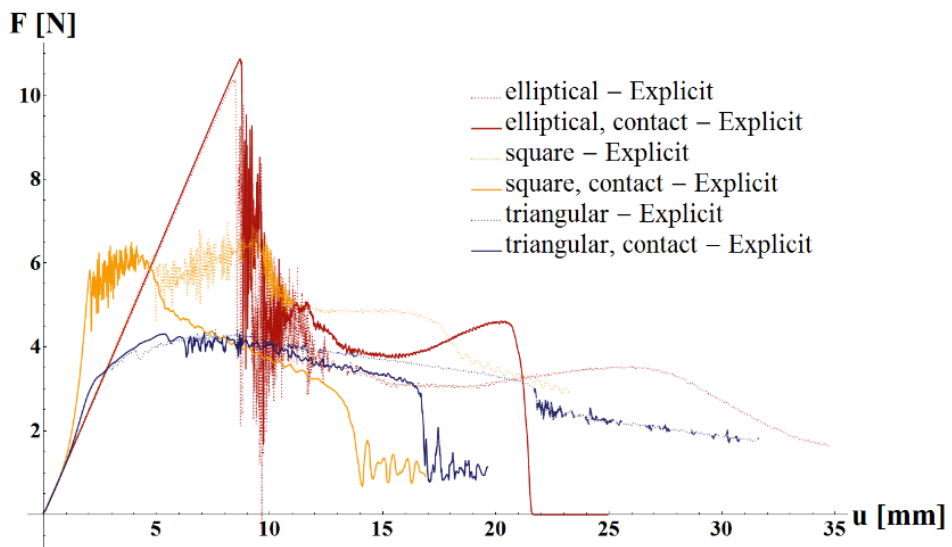

Fig. 8. Comparison of the reaction of the compressed bars with loading applied via contact - Explicit analysis.

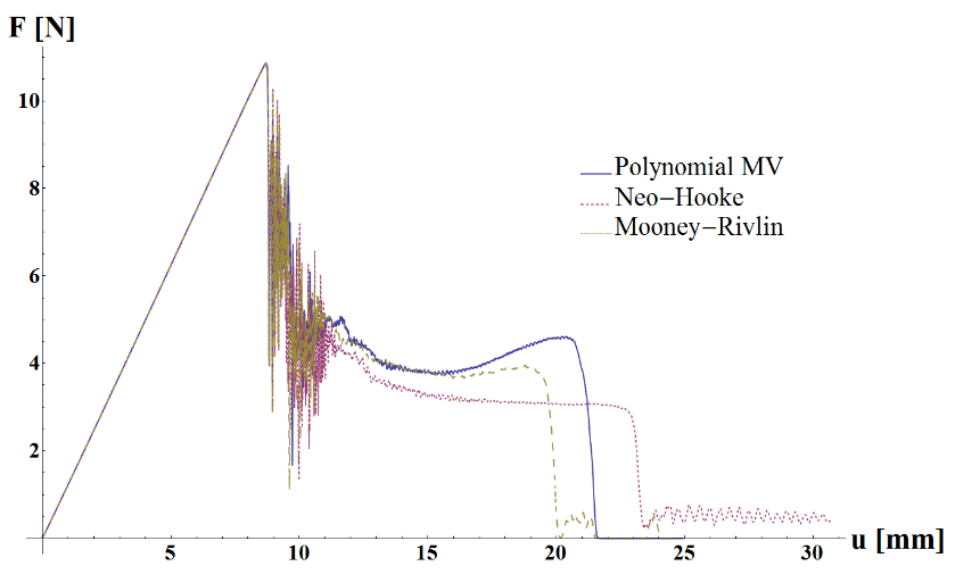

Fig. 9. Comparison of the compression reaction for the elliptical cross-section and different material models - Explicit analysis. 
Interesting phenomena can be observed when analysing the behaviour of the samples subjected to contact loading (Figure 8). The elliptical sample can be characterised by fairly similar response to the displacement loading case, however, the triangular and the square tube show significant raise in rigidity just before reaching the load capacity.

Table 2. Deformation history of the elliptical sample, LE - maximal logarithmic strain in sample (absolute value)

\begin{tabular}{|c|c|c|c|}
\hline $\mathrm{U}[\mathrm{mm}]$ & Deformation & $\begin{array}{l}\mathrm{LE} \\
\max \end{array}$ \\
\hline 8.5 & & 0.0359 \\
\hline 8.735 & & 0.0361 \\
\hline 8.755 & & 0.0753 \\
\hline 11.465 & & 0.0939 \\
\hline 11.470 & & & 0.1066 \\
\hline & & & \\
\hline
\end{tabular}

The only difference that can be observed between the compressed samples with different material models is the post-critical behaviour - which is shown in Figure 9, that compares the reaction of the models with contact boundary conditions.

Table 2 presents the exemplary deformation history of the elliptical sample analysed by ABAQUS/Standard, with displacement boundary conditions. The corresponding values in the table are: the displacement $U$ of the edge of the sample (load), deformation field with the map of the maximal absolute value of logarithmic strain LE, and the maximal value of LE in the whole model.

\section{Remarks and conclusions}

The work presents the analysis of local and global buckling of the rubber-like material tubes, comparing the results of models with different material constitutive relations [3], cross-sections and solved via different solution techniques. Three models of thin-walled pipes of the same stiffness with different cross-sections are considered. No imperfections are introduced in the FEM models of the analyzed pipes. Three types of constitutive models of a elastomeric incompressible material are used - with the polynomial function of elastic 
energy in the form of the model MV [3] and standard models of Neo-Hooke and MooneyRivlin [4]. Numerical simulations of buckling of pipes are performed for two types of initial-boundary value problems, i.e. quasi-static and dynamic ones. It was shown that the type of buckling (global or local) depends on the cross-section of the pipe.

While the ABAQUS/Standard program [1] allows to use the incompressible hyperelastic materials, the ABAQUS/Explicit program [2] does not have such a possibility, hence there is a need to define material parameters related to the spherical part of the stress tensor. In addition, the parameter should not be too small, as this can lead to numeric errors. The solutions of buckling of pipes modelling with different constitutive models are compered and good correlations of the results have been observed.

\section{References}

1. ABAQUS/Standard, User's manual, ver.6.11 (Dassault Systèmes, 2011)

2. ABAQUS/Explicit, User's manual, ver.6.11 (Dassault Systèmes, 2011)

3. S. Jemioło, Study of hyperelastic properties of isotropic materials. Modeling and numerical implementation. Scientific Works. Civil Engineering 14 (OWPW, Warszawa, 2002)

4. ABAQUS Theory Manual, ver.6.11 (Dassault Systèmes, 2011)

5. S. Jemioło, M. Gajewski, Hyperelastoplasticity (OWPW, Warszawa, 2017)

6. J. Bonet, R. D. Wood, Nonlinear continuum mechanics for finite element analysis. $2^{\text {nd }}$ edition (Cambridge University Press, Cambridge, 2008)

7. O. C. Zienkiewicz, R. L. Taylor, The finite element method. Volume 2. Solid mechanics. $5^{\text {th }}$ edition (Butterworth-Heinemann, 2000) 